\title{
Reverse Monte Carlo modeling in confined systems
}

\author{
V. Sánchez-Gil, E.G. Noya and E. Lomba \\ Instituto de Química Física Rocasolano, \\ CSIC, Serrano 119, E-28006 Madrid, Spain
}

(Dated: September 10, 2018)

\begin{abstract}
An extension of the well established Reverse Monte Carlo (RMC) method for modeling systems under close confinement has been developed. The method overcomes limitations induced by close confinement in systems such as fluids adsorbed in microporous materials. As a test of the method, we investigate a model system of ${ }^{36} \mathrm{Ar}$ adsorbed into two zeolites with significantly different pore sizes: Silicalite-I (a pure silica form of ZSM-5 zeolite, characterized by relatively narrow channels forming a 3D network) at partial and full loadings and siliceous Faujasite (which exhibits relatively wide channels and large cavities). The model systems are simulated using Grand Canonical Monte Carlo and, in each case, its structure factor is used as input for the proposed method, which shows a rapid convergence and yields an adsorbate microscopic structure in good agreement with that of the model system, even to the level of three body correlations, when these are induced by the confining media. The application to experimental systems is straightforward incorporating factors such as the experimental resolution and appropriate q-sampling, along the lines of previous experiences of RMC modeling of powder diffraction data including Bragg and diffuse scattering.
\end{abstract}

PACS numbers: 61.05.-a, 68.43.Fg 


\section{INTRODUCTION}

Neutron and X-ray scattering techniques have been for years useful tools to gain a better understanding of adsorption processes ${ }^{1-5}$, very specially in order to locate active sites and/or privileged positions for the adsorption of certain adsorbates. Given the small ratio between adsorbate/adsorbent molecules, and since in many instances the adsorbent exhibits a well defined crystalline structure, one can expect a diffraction pattern that will be dominated by long range order features. This situation recalls the problem of modeling powder diffraction data to account for lattice and magnetic disorder, which can be tackled by means of a Reverse Monte Carlo (RMC) approach by direct calculation of the structure factor ${ }^{6}$. As pointed out in Ref.6, the well established Rietveld refinement for modeling crystalline systems and its variants mostly concentrate on the Bragg scattering whereas local disorder -which gives rise to diffuse scattering- is not considered. In the case of adsorption in crystalline microporous materials, the adsorbate molecules do not necessarily exhibit crystalline order. The Rietveld refinement can be applied using hand-tuning to a certain degree when the number of adsorbate particles per unit cell is relative low (see Refs, 4 and 5 for examples of hydrocarbon adsorption in Silicalite-I), and it is the approach of choice whenever the adsorbate+adsorbent sample is fully crystalline, in which case the single crystal method can be used (see e.g. Refs. 7-10). This approach would be certainly impractical when there is a substantial degree of disorder.

In this work, we are interested in the elucidation of adsorbate structure in zeolites. These are materials with well defined microporous geometry, in which corner-sharing $\mathrm{AlO}_{4}$ and $\mathrm{SiO}_{4}$ tetrahedra form channels organized in 1D, 2D, and 3D networks accessible to different adsorbate molecules. The crystalline structure of standard zeolites is available from the literature ${ }^{11}$, and adsorbates will induce changes in the diffraction spectra due to either modifications in the symmetry of the system or to the presence of disorder. From the discussion in the preceding paragraph, it might seem that the RMC approach of Mellegård and McGreevy ${ }^{6}$, as implemented in the RMCPOW program ${ }^{12}$ could be suitable to elucidate the microscopic structure of adsorbates in the present instance. There are however, a few aspects that suggest that a different approach is needed. Firstly, in many cases, the changes induced in the zeolite framework structure induced by the adsorbate are negligible (see however Refs. 7 and 9 as examples in which relatively large adsorbates modify the spatial 
group of the adsorbate). This implies that a substantial contribution to the structure factor remains unchanged. On the other hand, if one tries to blindly implement the standard Monte Carlo moves of ordinary RMC approaches (basically molecular translations and/or rotations, or spin rotations to model magnetic disorder ${ }^{13}$ ) to molecules under tight confinement, most of the moves will be rejected, by which the efficiency of the procedure will be extremely poor as compared with that obtained in regular fluids and glasses. The nature of our problem strongly suggests that the standard translation/rotation moves must be complemented with particle creation/annihilation attempts that allow an efficient sampling. It comes to our aid, that standard adsorption volumetry experiments 14 provide with relative accuracy estimates of the number of adsorbed molecules per unit cell of the adsorbent. Bearing in mind this information, it is possible to construct an efficient Reverse Monte Carlo procedure that can recover the microscopic structure of the adsorbed fluid from powder diffraction spectra and adsorption volumetry experiments.

The aim of this work is to test the proposed approach, which we will denote by NReverse Monte Carlo (N-RMC) method for several model systems. The N-prefix underlies the fact that in this approach the number of particles, $\mathrm{N}$, is one of the variables to optimize. For our testing purposes, we have generated the structure factor of ${ }^{36} \mathrm{Ar}$ adsorbed in two different zeolites, namely, Silicalite-I and siliceous Faujasite, by means of Grand Canonical Monte Carlo (GCMC) simulations at different loadings. Those systems have been studied experimentally by Llewellyn and coworkers 2,15 and it is known that can reliably be modeled using GCMC simulations ${ }^{15,16}$. We will see how the proposed N-RMC approach, with the sole input of the relevant portion of the structure factor, the known zeolite structure, and an estimate of the number of adsorbate molecules per unit cell can accurately render the microscopic structure of the adsorbate in the course of a relatively short simulation run.

The rest of the paper is sketched as follows. The essentials of the method are introduced in Section [II. The most relevant results are commented upon in Section [II. Conclusions and future prospects are presented in Section IV 


\section{METHOD}

\section{A. Implementation of the Reverse Monte Carlo method under confinement}

As mentioned before, information about the microscopic structure of the adsorbed fluid can be obtained from neutron or X-ray powder diffraction measurements (see for example Ref. 2). In the case of neutron powder diffraction, we will be dealing with an orientationally averaged structure factor $\underline{6}$ :

$$
S(q)=\frac{2 \pi^{2}}{N V<b>^{2}} \sum_{\mathbf{q}^{\prime}}\left|F\left(\mathbf{q}^{\prime}\right)\right|^{2} \delta\left(q-q^{\prime}\right) / q^{\prime 2}
$$

where $N$ and $V$ are, respectively, the number of atoms and the volume of system (which in the case of a perfect crystal would reduce to those of the unit cell), $\mathbf{q}^{\prime}$ are the allowed vectors in the reciprocal cell, and $\langle b\rangle$ is the average of the coherent scattering lengths of the constituent atoms $b_{j}$. The $1 / q^{\prime 2}$ factor stems from the angular integration over all the possible $\mathbf{q}^{\prime}$ orientations in the powder sample ${ }^{6}$. Finally, $F(\mathbf{q})$ contains the correlations between the scattering nuclei and is given by:

$$
F(\mathbf{q})=\sum_{j=1}^{N} b_{j} \exp \left(i \mathbf{q} \mathbf{R}_{j}\right)
$$

where $\mathbf{R}_{j}$ denotes the position of the atom $j$ in the unit cell. When dealing with real experimental data, the $\delta$-function in Eq. (2.1) must be replaced by the instrument resolution function. As mentioned in Ref. 6 this can be any of the standard powder line shapes, e.g. a simple Gaussian distribution.

In many cases of interest the zeolite structure is hardly affected during the process of adsorption, and for practical purposes can be considered frozen. This is also a very common approximation in simulation studies ${ }^{17}$. Along these lines, in our calculation the positions of the zeolite constituent atoms will be kept frozen. Consequently, its contribution to the total structure factor remains constant during the RMC simulation. From an experimental point of view, one typically measures the structure factor of the sample with and without adsorbate. Since in our case, the zeolite structure is well known, we will be working with the difference structure factor,

$$
S_{\text {diff }}(q)=S_{\text {total }}(q)-S_{z e o-z e o}(q)
$$


where $S_{z e o-z e o}$ is assumed to correspond to the empty sample. From Eq. (2.1) the total structure factor can be calculated in our case using the following expression:

$$
S(q)=\frac{2 \pi^{2}}{N V<b>^{2}} \sum_{\mathbf{q}^{\prime}} \frac{1}{q^{\prime 2}}\left|\sum_{j=1}^{N_{z e o}+N_{a d}} b_{j} \exp \left(i \mathbf{q}^{\prime} \mathbf{R}_{j}\right)\right|^{2} \delta\left(q-q^{\prime}\right)
$$

where $N_{z e o}$ is the number of atoms of the zeolite and $N_{a d}$ is the number of adsorbed atoms. It is easy to see that the three partial contributions to the total structure factor can be calculated separately:

$$
\begin{gathered}
S_{z e o-z e o}(q)=\frac{2 \pi^{2}}{N V<b>^{2}} \sum_{\mathbf{q}^{\prime}} \frac{1}{q^{\prime 2}}\left|\sum_{j=1}^{N_{z e o o}} b_{j} \exp \left(i \mathbf{q}^{\prime} \mathbf{R}_{j}\right)\right|^{2} \delta\left(q-q^{\prime}\right) \\
S_{a d-a d}(q)=\frac{2 \pi^{2}}{N V<b>^{2}} \sum_{\mathbf{q}^{\prime}} \frac{1}{q^{\prime 2}}\left|\sum_{j=1}^{N_{a d}} b_{j} \exp \left(i \mathbf{q}^{\prime} \mathbf{R}_{j}\right)\right|^{2} \delta\left(q-q^{\prime}\right) \\
S_{z e o-a d}(q)=\frac{4 \pi^{2}}{N V<b>^{2}} \sum_{\mathbf{q}^{\prime}} \frac{1}{q^{\prime 2}}\left[\left(\sum_{j=1}^{N_{a d}} b_{j} \cos \left(\mathbf{q}^{\prime} \mathbf{R}_{j}\right)\right)\left(\sum_{j=1}^{N_{z e o}} b_{j} \cos \left(\mathbf{q}^{\prime} \mathbf{R}_{j}\right)\right)\right. \\
\left.+\left(\sum_{j=1}^{N_{a d}} b_{j} \sin \left(\mathbf{q}^{\prime} \mathbf{R}_{j}\right)\right)\left(\sum_{j=1}^{N_{z e o}} b_{j} \sin \left(\mathbf{q}^{\prime} \mathbf{R}_{j}\right)\right)\right] \delta\left(q-q^{\prime}\right)
\end{gathered}
$$

As mentioned, we will only calculate the relevant contribution $S_{\text {diff }}$-Eq.(2.3) - just adding Eqs.(2.6) and (2.7). Note, however, that in some cases the zeolite can undergo structural changes upon the adsorption of some molecules (usually big aromatic molecules $)^{\underline{7}-\underline{\underline{9}}}$. Obviously, in those cases the zeolite-zeolite contribution must be explicitly taken into account.

The core of the RMC method reduces to performing random particle moves that are accepted or rejected depending on whether the newly generated trial structure of the fluid (measured in terms of the pair distribution function, $g(r)$, or the structure factor, $S(q)$ ) approaches a target structure (usually an experimental $g(r)$ or $S(q)$ ). In the particular case that $S(q)$ is the reference property, the deviation from the target structure is measured using the statistical quantity,

$$
\chi^{2}=\sum_{i=1}^{N_{q}} \frac{\left(S_{\text {calc }}\left(q_{i}\right)-S_{\text {exp }}\left(q_{i}\right)\right)^{2}}{\sigma^{2}\left(q_{i}\right)}
$$

where the sum runs over the $N_{q}$ discrete values of the wave vector $q_{i}$ for which the structure factor $S(q)$ is evaluated, and $\sigma\left(q_{i}\right)$ is the standard deviation of $S_{\text {exp }}\left(q_{i}\right)$, that takes into account that experimental data carry different statistical uncertainties depending on the 
q-range of the measurements. In the standard RMC approach we will be dealing with translational or rotational trial movements. Following Ref. 18, the minimization of $\chi^{2}$ can be accomplished when the particle moves are accepted according to a probability given by

$$
P^{a c c}=\min \left(1, \exp \left(-\frac{\chi_{\text {new }}^{2}-\chi_{\text {old }}^{2}}{2}\right)\right)
$$

where $\chi_{\text {new }}$ and $\chi_{\text {old }}$ are the values of $\chi$ after and before the trial move. In common with other optimization techniques such as simulating annealing and standard canonical Monte Carlo (that minimizes the system's internal energy), moves that worsen $\chi^{2}$ can also be accepted as long as they comply with the probability distribution (2.9). In this way, the configurational space is adequately sampled and chances for the procedure to get trapped in local minima are greatly reduced.

Now, focusing on the problem of a system of tightly confined particles, as is the case of adsorbates in zeolite channels, an obvious problem with the scheme above described is the fact that most translational moves (and rotations in the case of molecules) will be rejected, due to overlaps with the zeolite framework. This means that, even if we are careful enough to generate an initial configuration of non-overlapping adsorbate molecules, the very low diffusivity within the channels and the high anisotropy of the medium, would render the standard RMC method inefficient. Our approach to speed up the sampling consists on starting from the empty zeolite and, in addition to the usual translational moves, also incorporate particle insertion and deletion trials. The number of sample particles can be estimated from a variety of experimental sources, for example, from volumetric adsorption experiments, and in standard RMC simulations is kept constant. In our approach, the acceptance rule is modified so that besides the minimization of $\chi^{2}$, a constraint on the target number of adsorbed particles, $N_{\text {exp }}$, is also included:

$$
P^{a c c}=\min \left(1, \exp \left(-\frac{\chi_{\text {new }}^{2}-\chi_{\text {old }}^{2}}{2}-\frac{\Delta N_{n e w}^{2}-\Delta N_{\text {old }}^{2}}{2}\right)\right),
$$

where $\Delta N^{2}=\left(N-N_{\text {exp }}\right)^{2} / \sigma_{N}^{2}, N$ being the instantaneous number of adsorbed particles and $\sigma_{N}$ the experimental uncertainty in $N_{\text {exp }}$.

In this work the target $S(q)$ and $N_{\text {exp }}$ will be obtained from GCMC simulations rather than from experiments and, therefore, both the target structure factor and the number of particles are accurately known. However, we would like to explore the effect of their uncertainties on the performance of the method. For that purpose we carried out simulations 
for several values of $\sigma_{N}$ and $\sigma\left(q_{i}\right)=\sigma_{S}, \forall i$. For simplicity, we have chosen an uniform value for the uncertainty of all q-values, but in real experiments this is not necessarily so. Note that the uncertainties play a similar role to the temperature in usual MC simulations, i.e. $\sigma_{S}$ and $\sigma_{N}$ control the equilibrium value and the magnitude of the allowed deviations of $\chi^{2}$ and $\Delta N^{2}$. The lower the value of $\sigma_{N}$ the better the quality of the fit, but also the smaller the fluctuations allowed in $\chi^{2}$; and the same applies to the number of particles $N$ depending on the value of $\sigma_{N}$.

Due to the strong confinement effect imposed by the zeolite, insertion and deletion moves are crucial to avoid the trapping of adsorbed atoms in particular regions of the zeolite and therefore, will play a key role to sample efficiently the configurational space. Additionally, the performance of the RMC in confined media can be much improved by imposing a bias in the insertion moves so that insertions are only attempted on those regions of the zeolite accessible to the adsorbate $\underline{\underline{19}}$. This is sufficient in our case (a monoatomic adsorbate), but when dealing with more complex adsorbates, such as chain or aromatic molecules, more sophisticated moves are needed. This is the same problem that one encounters in MC simulations of complex molecules in tightly confined media or at high densities. It can be tackled by using configurational bias moves ${ }^{20}$ that have been designed to greatly enhance the performance of sampling in the case of molecules with important steric constraints (see Refs. 19,21 for a comparison of the acceptance probability of purely random and various types of biased displacement/insertion schemes in MC simulations).

Finally, as usual in the RMC method one has to define an exclusion core around each of the sample particles. This core, that reflects the effective size of the particle, is needed in order to avoid unphysical overlaps, either between the adsorbates or between the adsorbed atoms and the zeolite framework. In our case, since we are dealing with model Lennard-Jones particles this quantity can be defined rather easily. Similar to the usual RMC other constraints can be applied, for example, a constraint on the adsorbate coordination number if many-body effects are known to be important ${ }^{22}$. In the examples studied here, many-body effects arise exclusively from the external field imposed from the zeolite rather than from adsorbateadsorbate interactions. The target structure factor was obtained from MC simulations in which Ar-Ar interactions are simple pairwise Lennard-Jones potentials. In the case of bulk systems interacting via pairwise potentials, it is known that the knowledge of the pair distribution function determines uniquely the pair potential 23 . Thus one should expect that 
in the particular instance of pairwise interacting systems reproducing the pair structure will guarantee an accurate representation of higher order correlation functions without further constraints in the RMC procedure. One must note however that in our case, effective manybody effects on the Ar-Ar correlations are at play through the external confining field. So the uniqueness of the structural resolution would be in question, except for the fact that in the case of zeolites the structure of the confining medium and its corresponding field are accurately known. With this in mind, there will be no need to impose extra constraints on the procedure, as it will be illustrated below.

\section{B. Simulation details}

As mentioned, in order to assess the validity of the N-RMC approach to study the structure of fluids under confinement, we have considered as test cases the adsorption of argon in two zeolites with significantly different pore sizes, namely, Silicalite-I that is formed by a network of straight and sinusoidal pores of diameter of about 5-5.5 $\AA$, and siliceous Faujasite that presents quite large cavities with diameters of about $11.5 \AA$. The first system was studied experimentally $\stackrel{2}{2}^{2}$ by means of adsorption and neutron scattering experiments. Nonetheless, for our test purposes, we find more convenient to generate the "experimental" structure factor from a GCMC simulation. In this way, the target structure is accurately known and we have access to all microscopic structural quantities of relevance to compare with 24 . Obviously this substantially simplifies the problem, removing the experimental data treatment from the picture, or the incorporation of the instrument resolution function (which should replace the $\delta$-function in Eq. (2.1)), and the appropriate treatment of the discrete sampling of q-space ${ }^{6}$. In our case we will be comparing $S(q)$ 's generated using identical simulation cells, by which all these subtleties can be omitted. Obviously, this will not be the case when dealing with real experimental data.

Explicitly, both in the GCMC and the N-RMC we have used a simulation box that contains $4 \times 4 \times 6$ unit cells of the orthorhombic Silicalite- $\mathrm{I}^{7}$ and $4 \times 4 \times 4$ for the Faujasite (see the structures of these zeolites in Figure 1). In the GCMC both the oxygen atoms in the zeolite and the argon atoms are modeled using Lennard-Jones interactions. The parameters of the LJ model were chosen from the bibliography ${ }^{25}$ and are given in Table I. Silicon atoms are surrounded by oxygen tetrahedra and therefore it is common not to assign a Lennard- 
Jones (LJ) center to them. We used periodic boundary conditions and the LJ potential was truncated at a distance of $13 \AA$. GCMC simulations of argon adsorption were performed at $77 \mathrm{~K}$ and in Silicalite-I at two different pressures that lead respectively to loadings of about 25.5 and 32 argon atoms per unit cell, the latter corresponding to saturation. In Faujasite the study was performed at a pressure corresponding to an intermediate loading of about 100 argon atoms per unit cell.

TABLE I: Parameters of the Lennard-Jones model used for the argon-argon and argon-zeolite interactions.

\begin{tabular}{ccc}
\hline \hline & $\epsilon / \mathrm{k}_{B}(\mathrm{~K})$ & $\sigma(\AA)$ \\
\hline Ar-Ar & 119.8 & 3.405 \\
$\mathrm{Ar}-\mathrm{O}$ & 117.2 & 3.121 \\
\hline \hline
\end{tabular}

The simulated structure factor (subtracting the zeolite-zeolite contribution) averaged over a GCMC simulation of about 100,000 MC cycles was used as the target in the N-RMC run. Here one cycle is defined as $N_{a d}$ particle translations attempts plus one insertion and one removal attempt. We have defined the particle size (or overlap distance) in the N-RMC as $\sigma_{\alpha \beta}(R M C)=0.92 \sigma_{\alpha \beta}$, (and $\sigma_{\alpha \beta}$ taken from Table I) taking into account that the distance of minimum approach of LJ particles is slightly less than the LJ $\sigma$ parameter.

During the RMC simulation we monitored the evolution of $\chi^{2}$ and the number of particles. As it can be seen in Fig. 2, at the beginning of the N-RMC run, the quantity $\chi^{2}$ drops very rapidly whereas the number of atom increases, until both quantities reach a plateau and finally oscillate around an average value. The magnitude of the oscillations in $\chi^{2}$ and $\Delta N^{2}$ can be controlled by the factors $\sigma_{S}$ and $\sigma_{N}$ that appear in the acceptance probability given in Eq. (2.10).

In addition to the straightforward comparison of the target and the RMC simulated $S(q)$ 's, in our case a better insight of the method's performance can be gained by inspecting the partial distribution functions and the three body correlation functions. The partial distributions are defined as:

$$
g_{\alpha \beta}(r)=\frac{n_{\alpha \beta}(r)}{\Delta V \rho_{\alpha}}
$$

where $n_{\alpha \beta}$ is the number of atoms of type $\beta$ at a distance between $r$ and $r+\Delta r$ of a central atom of type $\alpha, \Delta V$ is the volume of a spherical shell between $r$ and $r+\Delta r$, and $\rho_{\alpha}$ is the 
partial density of component $\alpha$. We have calculated the adsorbent-adsorbent (Ar-Ar) and the adsorbent-adsorbate (Ar-O) partial distribution functions. No particular information can be extracted from the correlations involving Si atoms, since they all are buried within the framework tetrahedra formed by the oxygen atoms. In order to investigate the three body correlations we calculated the bond angle distribution, which is defined as the integral of the three body correlation function $g^{(3)}\left(r_{1}, r_{2}, \cos \theta\right)$ over the first coordination shell:

$$
f(\theta)=16 \pi^{2} \int_{0}^{r_{c}} \int_{0}^{r_{c}} r_{13}^{2} d r_{13} r_{23}^{2} d r_{23} g\left(r_{12}\right) g^{(3)}\left(r_{13}, r_{23}, \cos \theta\right),
$$

where we chose $r_{c}$ as the position of the first minimum of the pair distribution function. This function gives the distribution of angles between pairs of nearest neighbors with respect to a central atom. In this case we restricted our study to the bond angle distribution for argon triplets. From a practical point of view, this quantity will be evaluated from the ensemble average of $\cos \theta_{132}$ histograms corresponding to the 132 triplets of particles which fulfill $r_{13}<r_{c}$ and $r_{23}<r_{c}$.

\section{RESULTS}

We will start presenting the results for argon adsorbed in Silicalite-I. For the case of a loading of about 25 molecules per unit cell and for the chosen values of the uncertainties, $\sigma_{S}$ and $\sigma_{N}$, the N-RMC runs were fully converged after $10^{7} \mathrm{MC}$ steps (see Fig. 2). Initially the number of particles increased rapidly until it reached the experimental value after about $6 \times 10^{5} \mathrm{MC}$ steps. Beyond this point the number of particles remains constant and particle insertion/deletion moves are no longer accepted. A potential enhancement of the algorithm would be the implementation of coupled insertion/deletion moves in which the former are guided by a cavity bias that takes into account the location of adsorbate molecules within the zeolite accessible volume. In the present instance this improvement has not been deemed necessary.

For the particular case studied here and for the chosen value of $\sigma_{N}$, the number of particles equilibrates exactly to $N_{\exp }$ for $\sigma_{N}=\sqrt{0.005} \approx 0.0707$. For larger values of $\sigma_{N}$ the final number of particles is different (although not too far) from the experimental value. The fact that even when the constraint on the number of particles is not included (which corresponds to the case of $\sigma_{N} \rightarrow \infty$ ) the final number of particles is relatively close to 
the experimental one is related to the high accuracy in $S(q)$. Note, however, that when using experimental data which are subject to larger uncertainties, the deviation from the experimental number of particles will be quite large unless $\sigma_{N}$ is given a value consistent with the experimental uncertainty. On the other hand, as mentioned before, the value of $\sigma_{S}$ was chosen as a compromise between the quality of the fit of $S(q)$ and an efficient sampling of the configurational space. Here we used $\sigma_{S} \approx \sqrt{\left(V\langle b\rangle^{2}\right) /\left(2 \pi^{2} \times 2 \times 10^{5}\right)}$. The effect of the choice of $\sigma_{S}$ will be discussed in more detail below.

The evolution of $\chi^{2}$ in the usual RMC method (which only includes displacement attempts) is also shown for comparison in Fig. 2. When using the usual RMC algorithm one needs a procedure to generate an initial configuration with the experimental number of adsorbed molecules, which can be obtained by random insertion of particles discarding those configurations that imply adsorbate-adsorbate or adsorbate-zeolite overlaps. In this work this procedure was accelerated by trying only insertions at positions of the zeolite accessible to the adsorbed particles. As it can be seen in Fig. 2, in this rather simple case that involves spherical particles and a moderate density of the adsorbed fluid, an initial configuration is obtained within about $5 \times 10^{5} \mathrm{MC}$ steps. For more complex molecules, such as for example long chain alkanes, more sophisticated bias algorithms that enhance the probability of insertion of particles will be needed to generate an initial configuration in a reasonable amount of time $\frac{19}{21}$. The RMC simulation started from this quasi-random configuration, which exhibits a quite large value of $\chi^{2}$, seems to be converging to the same value as the $\mathrm{N}-\mathrm{RMC}$ method although at a much lower pace. Indeed, after $1.5 \times 10^{7} \mathrm{MC}$ steps the RMC method has not reached equilibrium yet, the average value of $\chi^{2}$ still decreasing. The lower convergence of the RMC method can be attributed to the low diffusion of the particles in the zeolite. Note that the N-RMC method needs a slightly larger number of steps to reach the experimental number of particles than the random insertion method. However, the value of $\chi^{2}$ for the first configuration with $N_{\text {exp }}$ molecules in the N-RMC, although still quite high, is about two orders of magnitude lower than when particles are inserted randomly, which indicates that particles are distributed already in a configuration much closer to the experimental one. The fact that virtually no particle exchange moves are accepted beyond this point indicates that indeed much of the diffusion problems are overcome already in the filling process in the N-RMC. It is quite remarkable that even for a simple system as that studied here the N-RMC method speeds up the convergence considerably with respect to 
the usual RMC method. As mentioned before, when dealing with complex molecules the use of biased insertion/deletion moves is essential to sample efficiently the phase space. In that instance the advantages of a N-RMC approach with respect to a RMC method with simple translational/rotational moves (if the latter is feasible at all) should be more apparent.

In Figure 3, the structure factor $S_{\text {diff }}(q)$ and its separate argon-argon and argon-zeolite contributions obtained from the N-RMC and the target GCMC $S(q)$ 's for a loading of 25 argon atoms per unit cell are shown. The low-energy neutron scattering lengths have been taken from Ref. 26. Note that the spiky appearance of both the target and fitted $S(q)$ 's reflect the finite number of $q$-vectors sampled and that no experimental resolution function is taken into account. As mentioned before, all these factors must be explicitly incorporated in order to fit experimental data ${ }^{6}$. Along the N-RMC run, the number of particles rapidly converges to the experimental value (see Fig. 21), and the calculated and target $S(q)$ 's are hardly distinguishable, the relative difference between the GCMC and N-RMC lying usually below 1\% (obviously for very low intensity peaks relative errors can reach higher values, but this corresponds to very small absolute errors).

Besides the good quality of the fit of $S_{d i f f}(q)$, Figure 3 shows that the same applies to the fit of the partial structure factor. Larger relative differences between the target and calculated argon-argon partial structure stem from the very low intensity of certain peaks of little relevance, and are also an spurious result from the use of a constant $\sigma_{S}\left(q_{i}\right)$. The good quality of the calculated partial structure factors is an important result, since as both components (2.6) and (2.7) enter into $S_{\text {diff }}(q)$ some of their features could average out. Therefore a good agreement in $S_{\text {diff }}(q)$ does not necessarily imply the same for its partial components.

In order to get a better picture of the local order of the adsorbed fluid, and how this property is captured by the N-RMC approach, we analyze in Figures 4, 5and 6 the corresponding partial distribution functions extracted from both the N-RMC and the GCMC simulations at the two adsorbate loadings in Silicalite-I (as an example of tight confinement) and Faujasite (as an example of a more loose confinement). The bulk Ar distribution function evaluated at the same temperature and at zero pressure is also shown for comparison. For Silicalite-I, a first glance at the distribution functions shows that the adsorbed fluid is very structured compared to the homogenous fluid, exhibiting order over quite long distances, this order being induced by the topology of the zeolite channels. Note, however, that despite the long 
range of correlations observed in Figs. 4 and 5, the sole intense peak corresponds to the nearest neighbor shell, this peak being higher and narrower than in bulk Ar, as is typically the case for fluids confined in narrow porous systems. The second peak on the other hand is split in two, the splitting being more apparent for the higher loading. The remaining peaks have a much lower intensity, though they extend over a wider range of distances than in the bulk fluid. This is in marked contrast with the situation observed when dealing with much larger adsorbate molecules at high loading (see Ref.27), in which the adsorbed molecules are forced to occupy highly correlated positions in the framework channels, giving rise to much stronger interchannel adsorbate correlations. The argon-zeolite partial distribution functions are much less structured, reflecting the small ratio of Ar vs. oxygen atoms. In Faujasite, the larger size of the pores is reflected on a second fluid-like peak in the Ar-Ar distribution function that occurs at shorter distances than the second peak in the bulk case (see Fig. 6).

When comparing the N-RMC and GCMC partial distribution functions, the overall good agreement for both argon-argon and argon-oxygen correlations is readily apparent, both in Silicalite-I (at the two loadings) and in Faujasite. The small differences on the first peak of Ar-Ar distribution function arise due to the finite size of the simulation box. An accurate reproduction of the short- $r$ behavior of $g(r)$ requires to have a detailed and accurate knowledge of the large $q$ behavior of $S(q)$. This in turn implies both the use of a large system size that allows a finer sampling of $q$-space and the inclusion of a rather long $q$-range in the fitting procedure.

Discrepancies in the first peak are more evident in Silicalite-I, i.e., in the system with smaller pores that imposes a tighter confinement. This is not unexpected, a closer confinement leads to a more solid-like behavior of the adsorbed fluid and relevant features in the $S(q)$ extend to larger $q$ than in systems with a more fluid-like behavior. Obviously a small uncertainty in $S(q), \sigma_{S}$ in Eq. 2.10, is also required to accurately reproduce the first peak in the partial distribution functions. As shown in Figure $7, \chi^{2}$ equilibrates to a lower value by decreasing $\sigma_{S}$, which means that the N-RMC $S(q)$ is closer to the target $S(q)$. As mentioned before, we chose a value that allowed us to sample the configurational space in a reasonable amount of time and at the same time produces a fairly good quality fit. We checked that the chosen value corresponds to a very small relative error of about $0.001 \%$ for the most intense peak, whereas the relative error grows up to $1 \%$ for peaks with an intensity a thou- 
sand times smaller than the most intense peak. Experimental data usually have a larger statistical uncertainty of about a few percent (even in the more intense peaks). We checked the effect of a relative error on the target structure factor and found that the distribution function was still reproduced with a very good accuracy. If any kind of medium-long range order builds up in the adsorbate within the zeolite, N-RMC should be able to provide an appropriate microscopic picture of it in consonance with the quality of the experimental data.

Further insight into the structure of the adsorbed fluid can be gained from the angular distribution function for triplets of argon atoms (see Figure 8 for Silicalite-I and Figure 9 for Faujasite). As mentioned before, we integrated the triplet correlation function up to the first minimum in the argon-argon partial distribution function $(\approx 5 \AA)$. For Silicalite-I the bond angle distribution is similar at moderate and at high loadings, exhibiting peaks at roughly the same angles, but, as expected, the peaks are sharper at a high loading as a consequence of the higher density and reduced mobility of the adsorbed atoms. The two peaks at high angles reflect the tendency of the argon atoms to adopt local linear configurations imposed by the confinement in the channels of the zeolite. The GCMC and N-RMC bond angle distribution functions agree very well at both loadings except for some small discrepancies in the strong peak at short angles (these differences being connected to the small error in the first peak of the Ar-Ar pair distribution function). On the other hand, the bond angle distribution of Ar in Faujasite is more similar to the bulk LJ fluid, reflecting the larger pores in this zeolite. In this case the agreement between the GCMC and the N-RMC bond angle distribution is almost perfect. The correct prediction of the three body distribution function obtained here indicates that the RMC method is able to capture the three-body correlations induced mostly by the external field created by the confining medium. In our particular case the structure of the confining medium is accurately known. Obviously, in those instances where the intermolecular interactions of the adsorbate are strongly directional with a significant influence of three body forces (e.g. in the case of zeolite templated carbons ${ }^{28}$ ) additional constraints must be imposed along the RMC procedure, as it is customary in many RMC applications (see for instance Refs. 29,30 for particular applications to disordered carbon materials). In any case, as shown for diatomic molecules and water, results obtained from RMC simulations need to be always interpreted with caution, as the correct description of the pair distribution function in real systems does not necessarily mean a good reproduction 
of the higher order distribution functions ${ }^{31}, 32$. Nonetheless, as explained before, for tightly confined media, one would expect that geometric effects play a more significant role. In those instances, the N-RMC approach can be a very useful tool.

Before concluding we would like to comment on the range of $q$ used to fit the $S(q)$. For the example presented here choosing a rather narrow range of $q(q \leq 5 \AA)$ was shown to be enough to obtain a very good description of the adsorbed fluid. Indeed, simulations including a broader range of $q$ did not lead to a substantial improvement of the results. An appropriate sampling of the reciprocal space (by increasing the simulation box more reciprocal vectors are included in the evaluation of $\mathrm{S}(\mathrm{q})$ ) has a higher impact on the quality of the results. The reason why including a higher range of $q$ has little influence on the results is that oscillations in the target $S(q)$ practically die out for $q>5 \AA$ (see Fig. 3). This can be understood as the result of the relatively simple short range structure of the adsorbed Ar, which must be recalled is one of the simplest fluids in all respects. However, for systems in which the short range $g(r)$ displays significant features (e.g. due to intramolecular correlations), fine long range details of $S(q)$ cannot be neglected, and consequently a broader range of $q$ must be included in the fitting procedure. When dealing with real systems, these data can be obtained from x-ray or neutron diffraction experiments, which currently allow to acquire fairly high resolution data up to quite large values of $q$.

\section{CONCLUSIONS}

In summary, we have presented a simple extension of the Reverse Monte Carlo method that enables the determination of the microscopic structure of fluids under confinement. The success of our test case study of a monoatomic fluid adsorbed into two well known zeolites (Silicalite-I and Faujasite), evidences the performance of the proposed method. Our approach can easily be extended to other systems, even disordered porous materials, provided a previous study to determine the structure of the adsorbent material is performed as prerequisite. Complex molecular adsorbates can also be dealt with resorting to bias sampling techniques. In a forthcoming publication we will demonstrate the application of the method to determine the structure of adsorbed aromatic hydrocarbons in various zeolites using both X-ray diffraction data and volumetric and microcalorimetric adsorption experiments as input for the N-RMC. 


\section{Acknowledgments}

The authors gratefully acknowledge the support from the Spanish MINECO (Ministry of Innovation and Economy) grant No. FIS2010-15502 and from the Autonomous Community of Madrid under Grant No. S2009/ESP/1691. The CSIC is also acknowledged for providing support in the form of the project PIE (Proyecto Intramural Especial) 201080E120. V.S.G. also acknowledges the CSIC for support of his work by means of a JAE program $\mathrm{PhD}$ fellowship.

\section{References}

1 P. L. Llewellyn, J. P. Coulomb, Y. Grillet, J. Patarin, G. Andre, and J. Rouquerol, Langmuir 9, $1852-1856$ (1993).

2 P. L. Llewellyn, J. P. Coulomb, Y. Grillet, J. Patarin, H. Lauter, H. Reichert, and J. Rouquerol, Langmuir 9, 1846 - 1851 (1993).

3 J. Coulomb, P. Llewellyn, Y. Grillet, and J. Rouquerol, Stud. Surf. Sci. Catal. 87, 535 (1994).

4 N. Floquet, J. P. Coulomb, G. Weber, O. Bertrand, and J. P. Bellat, J. Phys. Chem. C 107, 685 (2003).

5 N. Floquet, J. P. Coulomb, J. P. Bellat, J. M. Simon, G. Weber, and G. Andre, J. Phys. Chem. C 111, $18182-18188$ (2007).

6 A. Mellergård and R. L. McGreevy, Acta Crys. A 55, 783 (1999).

7 H. van Koningsveld, H. V. Bekkum, and J. C. Jansen, Acta Cryst. B 43, 127 (1987).

8 H. van Koningsveld, J. Jansen, and H. van Bekkum, Zeolites 10, 235 (1990).

9 H. van Koningsveld, F. Tuinstra, H. van Bekkum, and J. C. Jansen, Acta Cryst. B45, 423 (1989).

10 N. Kamiya, W. Iwama, T. Kudo, T. Nasuno, S. Fujiyama, K. Nishi, and Y. Yokomori, Acta Crys. B 67, 508 (2011).

11 C. Baerlocher, L. McCusker, and D. Olson, Atlas of Zeolite Framework Types (Elsevier, Amsterdam, The Netherlands, 2007), 6th ed. 
12 A. Mellergaard and R. McGreevy, Chemical Physics 261, 267 (2000).

13 A. Mellergård, R. L. McGreevy, A. Wannberg, and B. Trostell, J. Phys.: Condens. Matter 10, 9401 (1998).

14 S. Gregg and K. Sing, Adsorption, Surface Area and Porosity (Academic, London, 1982).

15 G. Maurin, R. Bell, B. Kuchta, T. Poyet, and P. Llewellyn, Adsorption 11, 331 (2005).

16 R. J. Pellenq and D. Nicholson, Langmuir 11, 1626 - 1635 (1995).

17 B. Smit and T. L. M. Maesen, Chem. Rev. 108, 4125 (2008).

18 R. L. McGreevy and L. Pusztai, Mol. Simul. 1, 359 (1988).

19 R. Q. Snurr, A. T. Bell, and D. N. Theodorou, J. Phys. Chem. 97, 13742 - 13752 (1993).

20 D. Frenkel and B. Smit, Undesrtanding Molecular Simulation (Academic, London, 2002).

21 J. I. Siepmann and D. Frenkel, Mol. Phys. 75, 59 (1992).

22 R. L. McGreevy, J. Phys.: Condens. Matter 13, R877 (2001).

23 R.L. Henderson, Phys. Lett. 49 A, 197 (1974).

24 L. Pusztai, and D. Tóth, J. Chem. Phys. 94, 3042 (1991).

25 M. D. Macedonia, D. D. Moore, and E. J. Maginn, Langmuir 16, 3823 (2000).

26 S. W. Lovesey, Theory of Neutron Scattering from Condensed Matter, vol. 1 (Clarendon Press, Oxford, England, 1987).

27 R. Marguta, S. Khatib, J. Guil, E. Lomba, E. Noya, J. Perdigón-Melón, and S. Valencia, Microporous and Mesoporous Materials 142, 258 (2011).

28 H. Nishihara, Q.-H. Yang, P.-X. Hou, M. Unno, S. Yamauchi, R. Saito, J. I. Paredes, A. Martínez-Alonso, J. M. Tascón, Y. Sato, M. Terauchi, and T. Kyotani, Carbon 47, 1220 -1230 (2009).

29 P. Zetterström, S. Urbonaite, F. Lindberg, R. G. Delaplane, J. Leis, and G. Svensson, J. Phys.: Condens. Matter 17, 3509 (2005).

30 N. A. Katcho, P. Zetterström, E. Lomba, J. F. Marco, E. Urones-Garrote, D. Avila-Brande, A. Gómez-Herrero, L. C. Otero-Díaz, and A. R. Landa-Cánovas, Phys. Rev. B 77, 195402 (2008).

31 A. De Santis, and D. Rocca Mol. Simul. 17, 143 (1996).

32 P. Jedlovszky, I. Bakó, G. Pálinkás, T. Radnai, and A. K. Soper J. Chem. Phys 105, 245 (1996). 
(a)
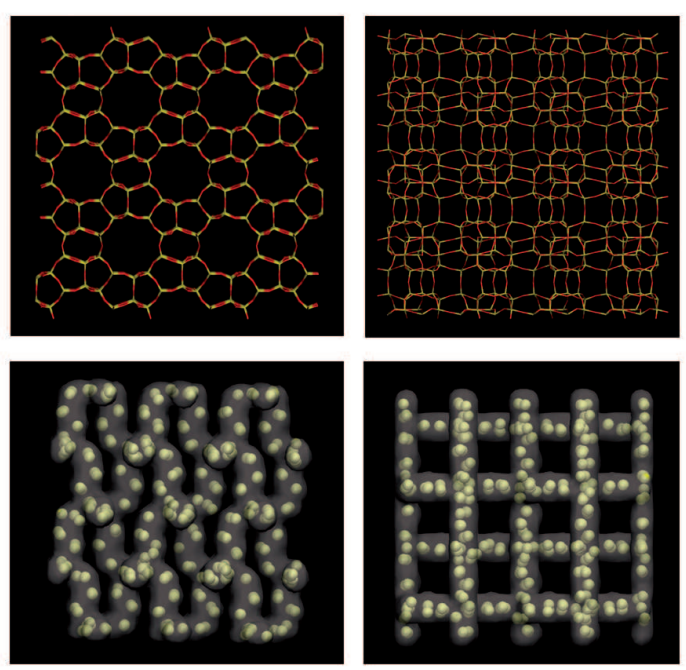

(b)
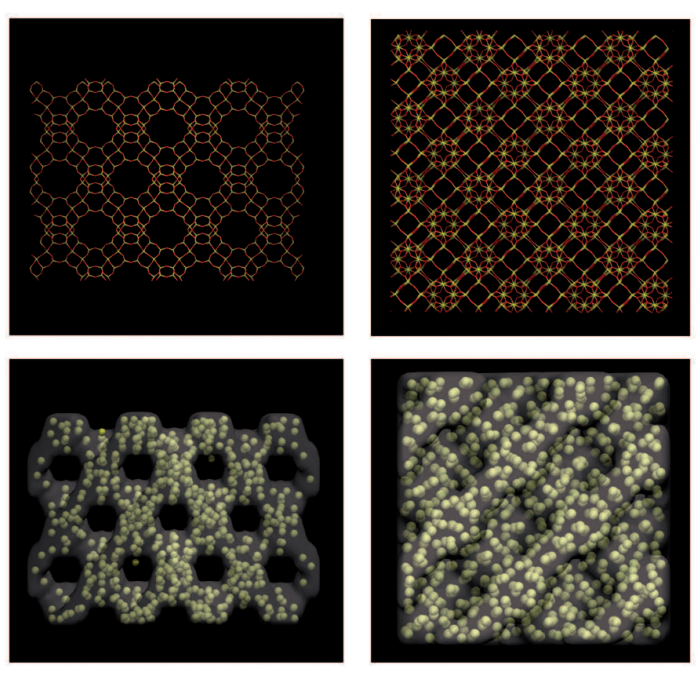

FIG. 1: Structure of the two zeolites considered in this work: (a) Silicalite-I and (b) Faujasite. In each case, the two top panels show two different views of the zeolite structure and the two bottom panels show the volume of the channels in a grey shadow and the adsorbed molecules (at a loading of 32 atoms per unit cell in the case of Silicalite-I, and 100 atoms per unit cell in the case of Faujasite). For clarity purposes this figure shows smaller systems that the one simulated in this work. 

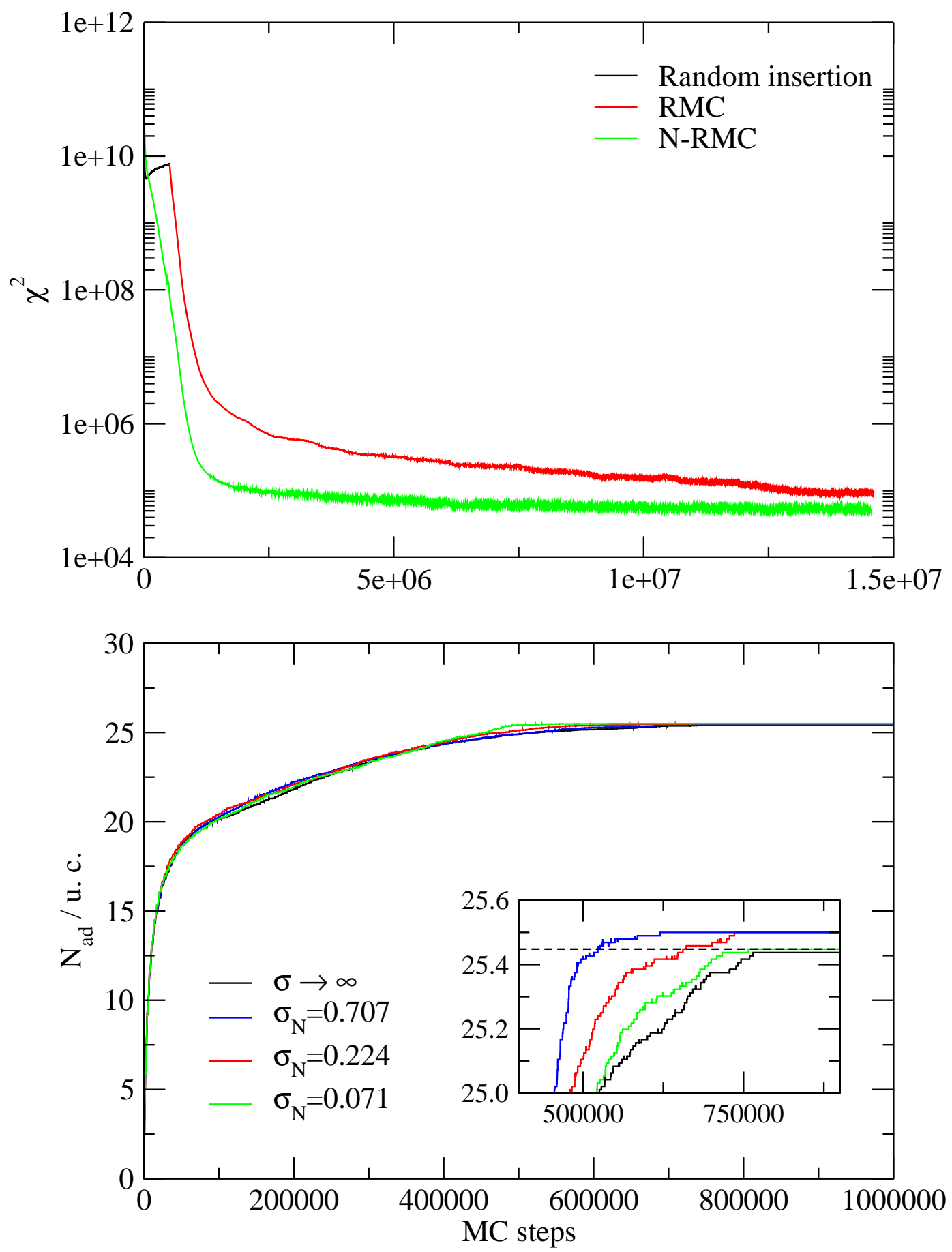

FIG. 2: Evolution of $\chi^{2}$ and the number of adsorbed particles $N_{a d}$ per unit cell along the N-RMC simulation. The evolution of $\chi^{2}$ with the usual RMC code is also shown for comparison. The black line shows the value of $\chi^{2}$ during the random insertion of molecules used to generate the initial configuration for the usual RMC algorithm. Along with the evolution of the number of particles for the value of $\sigma_{N}$ used in this work (shown in green), the results for other values of $\sigma_{N}$ are also shown. The dashed black line in the inset shows the target $N_{\text {exp }}$. 


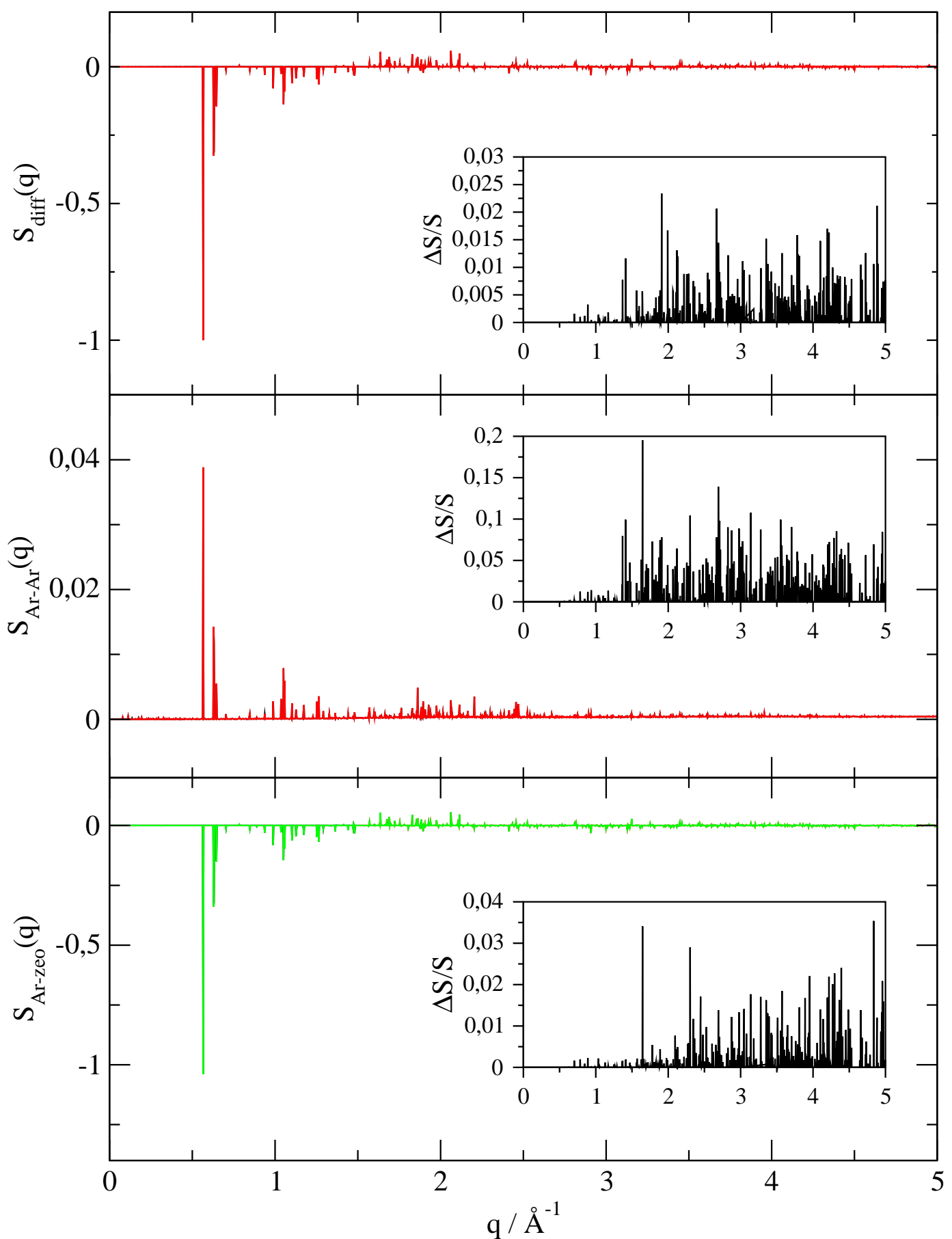

FIG. 3: Comparison of the target GCMC (black line) structure factor and that obtained from the N-RMC (red line) in Silicalite-I at a loading of about $25.5{ }^{36} \mathrm{Ar}$ atoms per unit cell. Note that the zeolite-zeolite partial structure factor has been subtracted so that only the sum of the argon-argon and argon-zeolite partial structure factors are used in the fit. The two separate partial structure factors are also shown. The insets show the relative difference between the GCMC and N-RMC structure factors. 


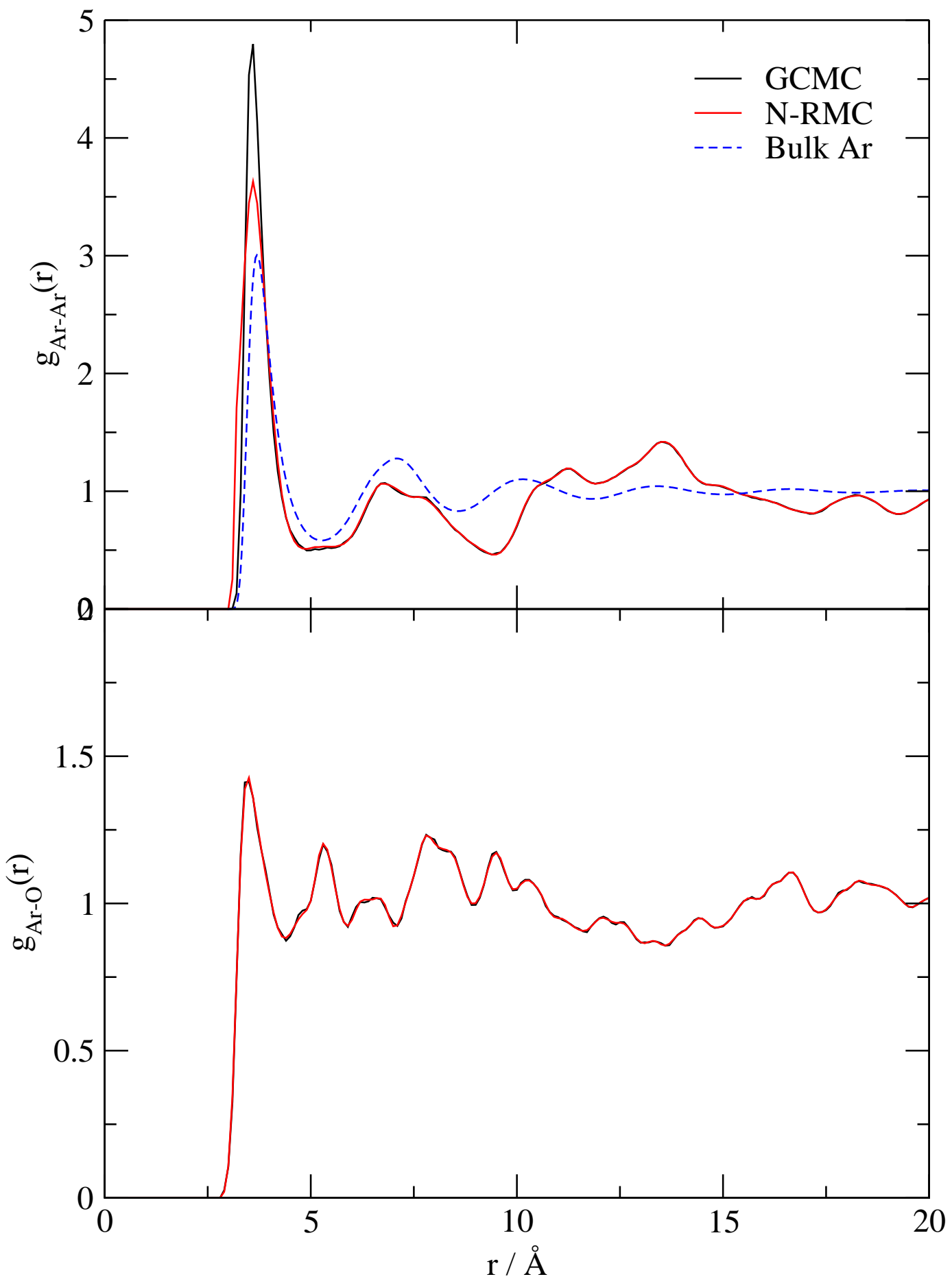

FIG. 4: Comparison of the target (GCMC) and the N-RMC partial Ar-Ar and Ar-O pair distribution functions in Silicalite-I at a loading of 25.5 atoms per unit cell. 


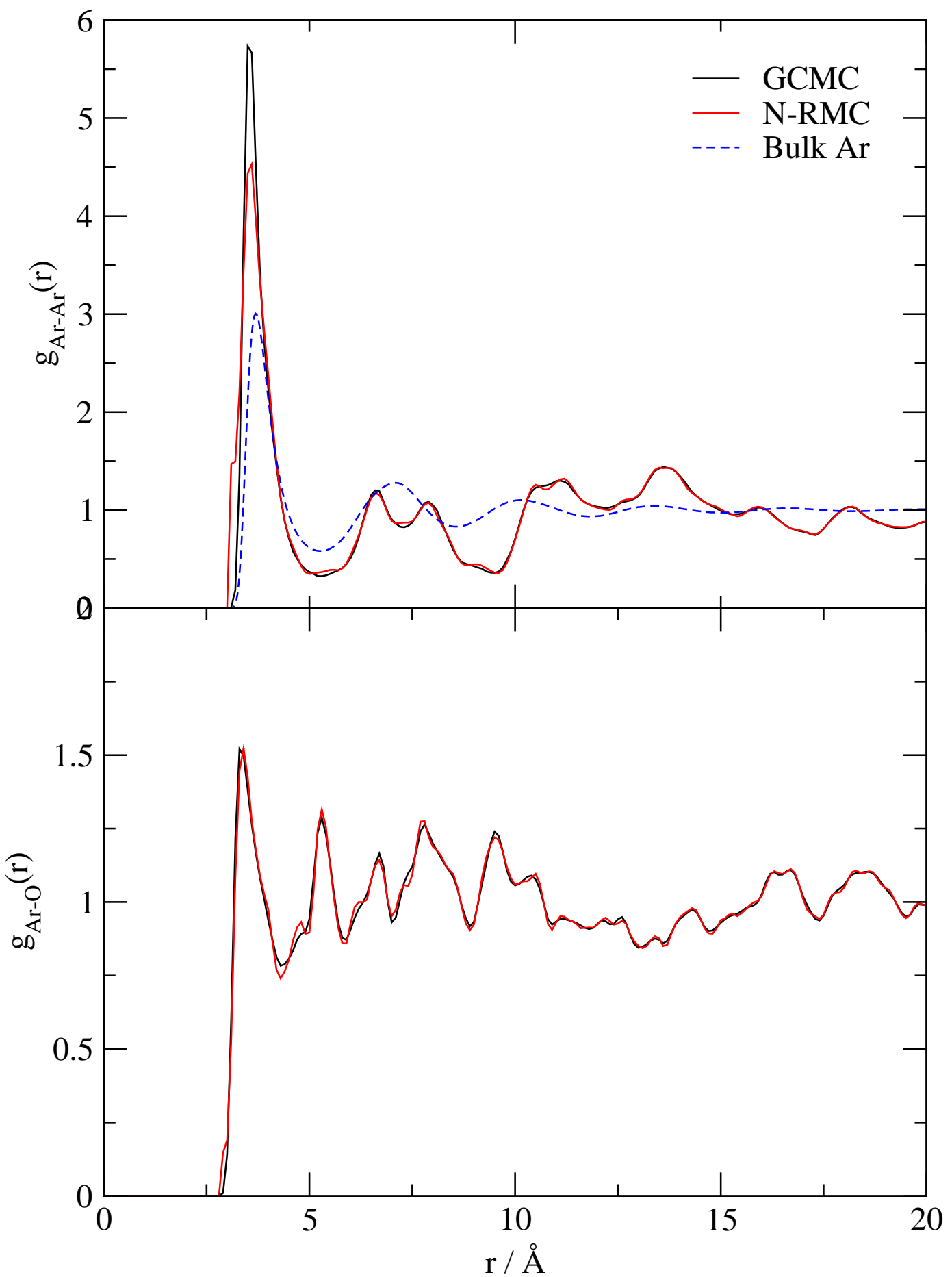

FIG. 5: Comparison of the target (GCMC) and the N-RMC partial Ar-Ar and Ar-O pair distribution functions in Silicalite-I at a loading of 32 atoms per unit cell. 


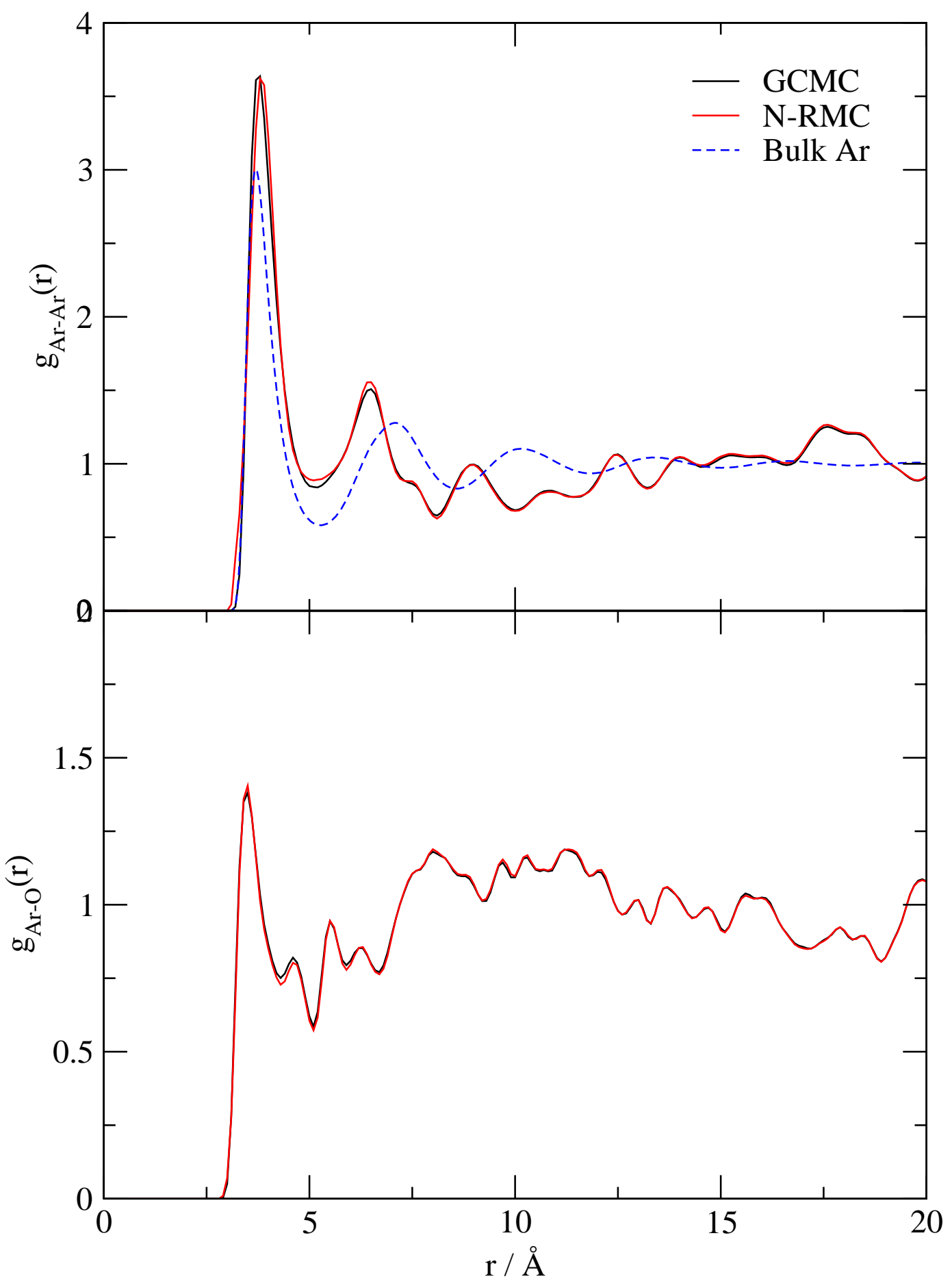

FIG. 6: Comparison of the target (GCMC) and the N-RMC partial Ar-Ar and Ar-O pair distribution functions in Faujasite at a loading of about 100 atoms per unit cell. 

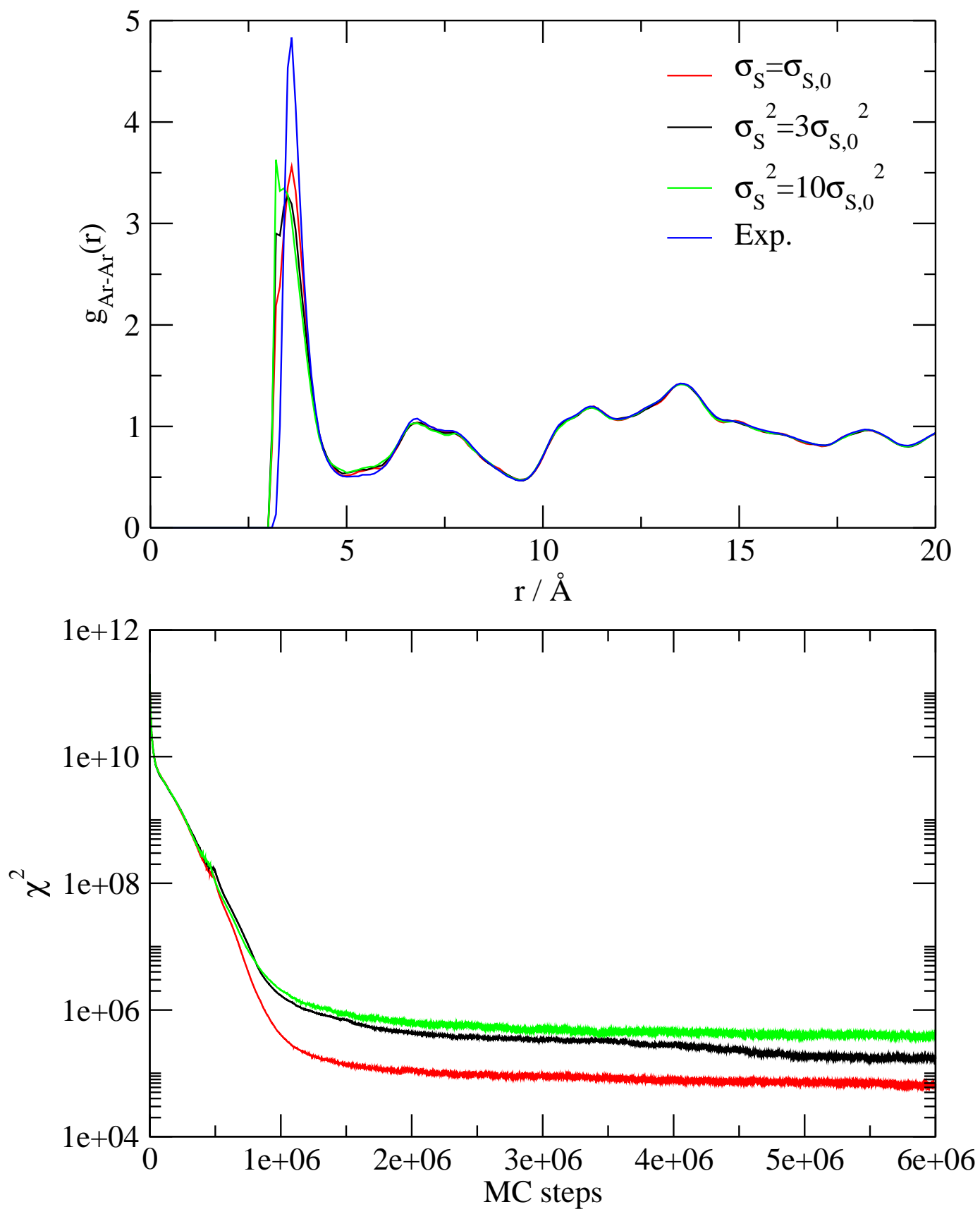

FIG. 7: Effect of the $\sigma_{S}$ parameter on the resulting pair distribution function (upper panel) and on the evolution of $\chi^{2}$ with the MC step (lower panel) in the case of a loading of 25.5 atoms per unit cell in Silicalite-I. $\sigma_{S, 0}$ corresponds to the value used in this work, $\sigma_{S, 0}=\sqrt{\left(V\langle b\rangle^{2}\right) /\left(2 \pi^{2} \times 2 \times 10^{5}\right)}$. 


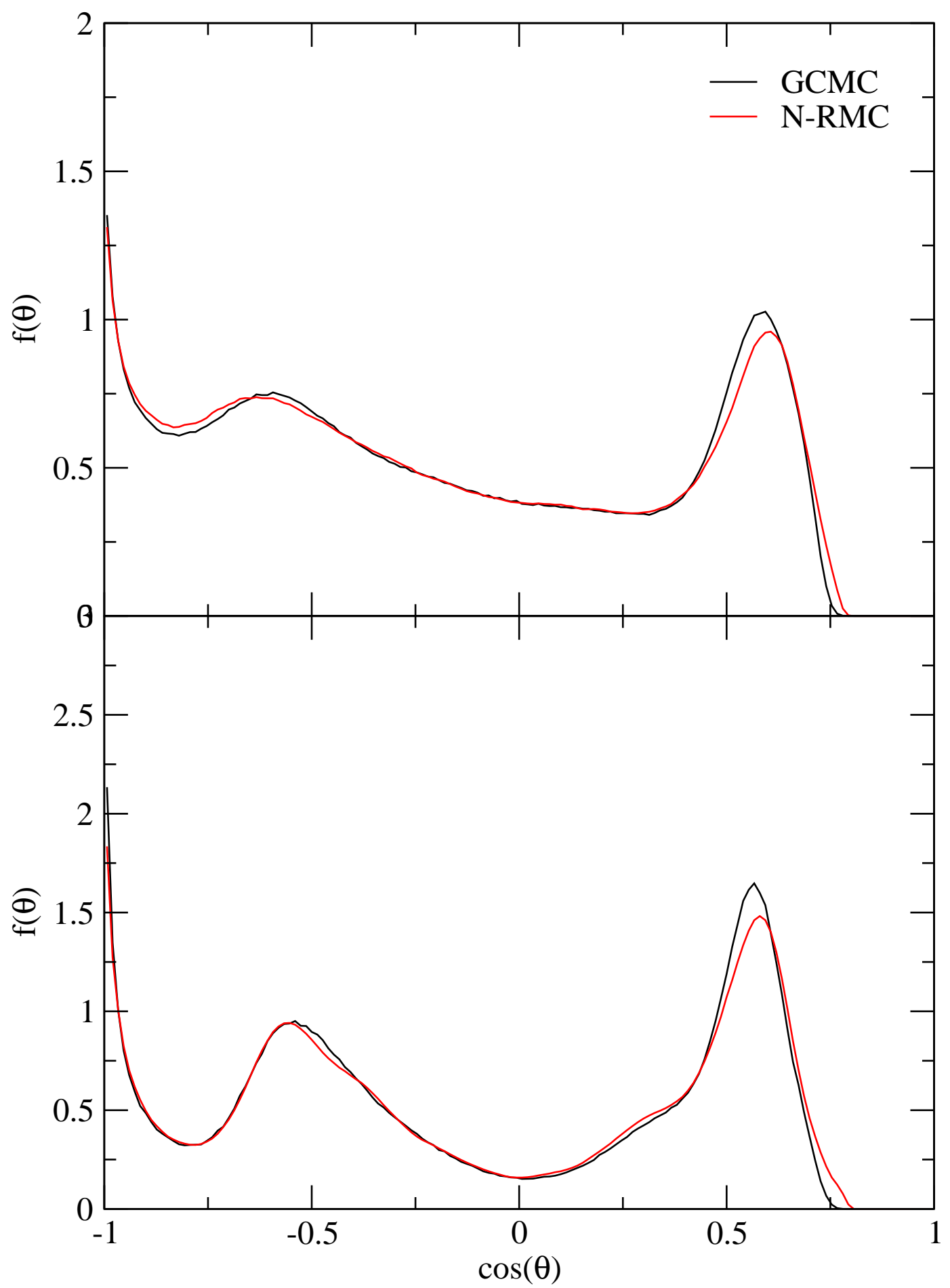

FIG. 8: Comparison of the target (GCMC) and the N-RMC bond angle distribution function Eq.(2.12) - for argon triplets at a loading of about 25.5 atoms per unit cell (top panel) and 32 atoms per unit cell (bottom panel) in Silicalite-I. 


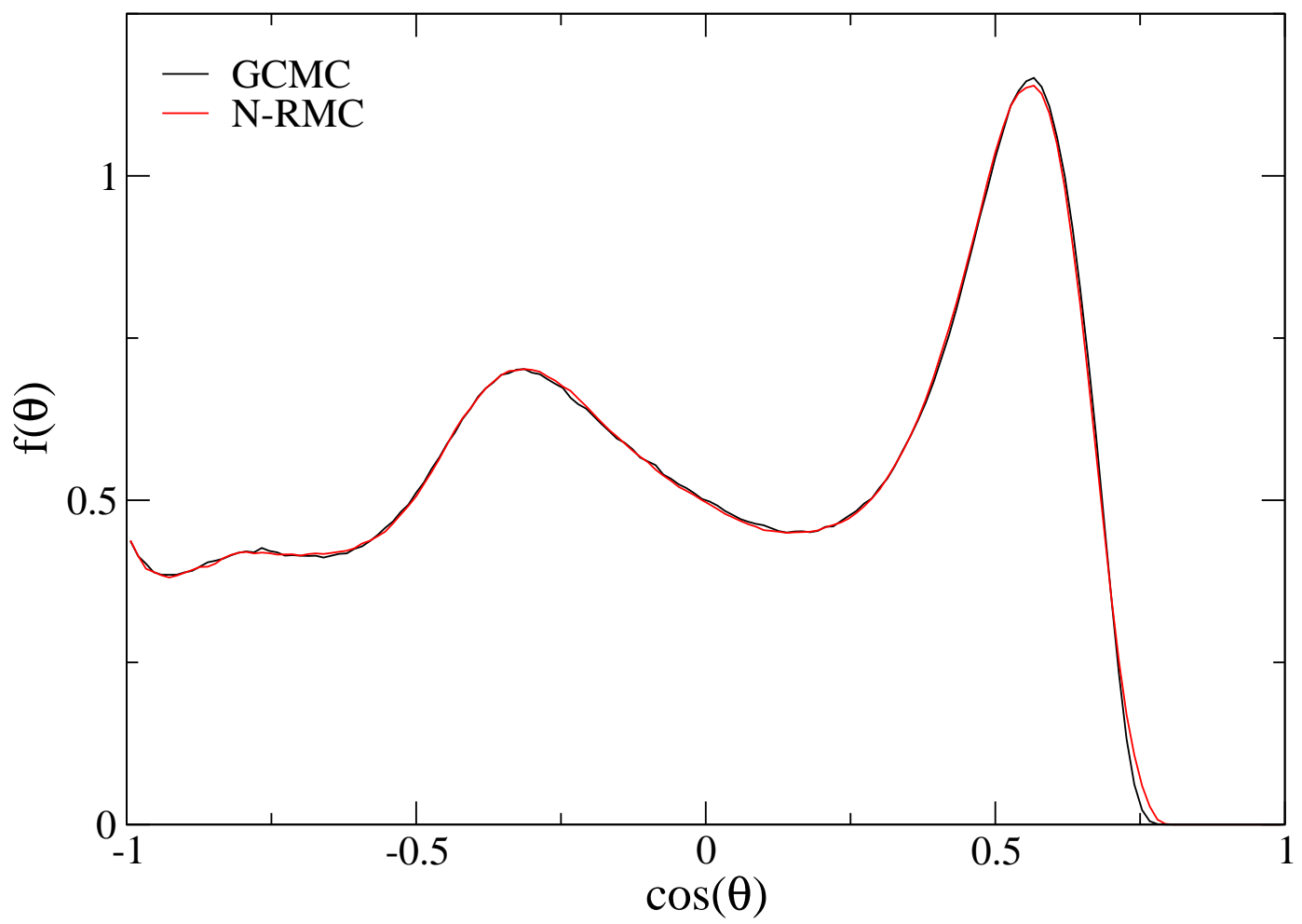

FIG. 9: Comparison of the target (GCMC) and the N-RMC bond angle distribution function -Eq.(2.12) - for argon triplets at a loading of about 100 atoms per unit cell in Faujasite. 